\title{
IDETC2016-59201
}

\section{ADVANCES IN BALANCE AND BIOFEEDBACK MEASUREMENT: THE CASE FOR HEALTH-BASED, POSTURAL SERIOUS GAMES}

\author{
Theodore Lim \\ School of Engineering \& Physical Sciences \\ Heriot Watt University, Edinburgh, UK
}

\author{
Daniil Yurchenko \\ School of Engineering \& Physical Sciences \\ Heriot Watt University, Edinburgh, UK
}

\author{
Gnanathusharan Rajendran \\ School of Life Sciences \\ Heriot Watt University, Edinburgh, UK
}

\author{
Aparajithan Sivanathan \\ School of Engineering \& Physical \\ Sciences \\ Heriot Watt University, Edinburgh, UK
}

\author{
James Ritchie \\ School of Engineering \& Physical \\ Sciences \\ Heriot Watt University, Edinburgh, UK
}

\begin{abstract}
Health games are increasingly seen as a means to address issues from therapy and rehabilitation. Yet, as a transformative technology, rarely have such games been explored or exploited to assist research into pathologies. Serious games for research (SGR) to uncover pathologies would allow clinicians to develop new differential diagnostics while providing a positive experience for the subject. This paper is not about game design; nevertheless it presents an outlook to considerations that could be taken forward when developing health-based SGRs for pathomechanics, etiopathogenesis and biofeedback. This work relates to preliminary studies on balance challenges manifested in pathologies of the central nervous system. As technology advancements seek to augment human sensory contact between virtual and real worlds this may impact on how virtual environments are used and designed in future. As a consequence heightened sensory (or lack of thereof) may result in falls, for example users with vestibular disorder - because postural stability is a key aspect of motor ability that allows individuals to sustain and maintain the desired physical position of their body Here, our investigation is specific to functional correspondence of the incidental properties in human body sway between healthy subjects and subjects with dyslexia. Our early results suggest postural sway between healthy subjects and those with mild disorders can be distinguished.
\end{abstract}

\section{INTRODUCTION}

The human body is an inherently unstable system [1]. Without our motor system continuingly correcting for imbalance, one will be at risk of falling. Research indicates neuro-musculoskeletal disorders relate to some degeneration in human balance [2] and are not simply a normality of aging. The human central nervous system is highly adept at compensating, and until deprived temporarily, a loss function due to the pathology may not become apparent. Human balance, postural control during standing and walking rely on "working memory" [3]. Intrinsically linked to motor development are biologically significant activities of posture maintenance and locomotion [4], [5] that concerns the learning of new motor skills [6]. Recent studies also show posture can influence cognitive development in infants [7].

To date, how humans maintain balance is not fully understood. In particular, balance disorders due to neuro-related pathologies remain open [8]. Understanding how the sensory, nervous and motor system work to generate compensation strategies and quantifying its status at any point in time could reveal underlying pathophysiological mechanisms and deficits.

Over the years, digital games have increasingly been deployed as an alternative to tackle serious issues. Health games largely remain focused on improving physical and mental health, well-being, adherence to treatment regiments and patient management. However, health games for research could help uncover pathological disorders, such as those associated with human balance. Being more narrowly focused and designed to achieve individual-level goals these new game modes are ideally suited to exploit specific programs or tools for meta-analysis and it is in this aspect that the reported work is aligned.

The initiative begins by investigating balance challenges for users in virtual environments (e.g. digital games) who may have a pathophysiological deficit. The aim is to gain a better understanding of the implications both at the level of the virtual 
environment as well as the sensory awareness of internal bodily and cognitive processes, while at the same time understand the design requirements to serendipitously serve emergent functions. This preliminary work examines postural instability due to sway in healthy subjects and subjects with dyslexia. Sway characteristics in human postural control has received limited investigation in relation to the onset of neurological disorders. The long term objective is to cross-correlate stabilograms and electroencephalography (EEG) as a new means to diagnose central nervous system pathologies.

\section{BACKGROUND AND IMPETUS}

Neural activities associated to sway characteristics in human postural control have received little attention with respect to cross-modal correlation of balance with respect to pathophysiological mechanisms and deficits. Although a simple task, quiet standing still requires cognitive resources [9], [11][18]. Balance is the result of interplay between complex control systems mediated by different brain pathways. Quiet stance experiments conducted using force-plate measurements are well reported in literature [11]-[18]. Posturographic studies have found significant differences in balance of Parkinson's disease patients. However, the method needs few improvements to diagnose individual patients [19]. Studies also show a significantly greater sway area for children with autism spectrum disorders [20]. The frequencies of the oscillation of the centre of pressure (COP) are lower than $5 \mathrm{~Hz}$ [21].

Neurological defects have been investigated through EEG data in subjects with neurological defects such as dyslexia and dysgraphia among others [22]-[24], as well as the neurological relationship with balance. There is a body of literature based on muscle movement [25], including indirect balance properties such as power spectral density of sway in males [26], and the modelling of the control of human upright balance [27]. Early research on healthy subjects by Kruse et al [28] suggests cortical EEG rhythms might indicate a class of neuromuscular work; alpha range are associated to cortical processes regulating neuromuscular processes, while theta range associate with reflexes and synergies that permit balance and stable body positioning. Hari and Salmelin [29] suggest that a $10 \mathrm{~Hz}$ rhythm is predominantly a somatosensory cortical function, and the $20 \mathrm{~Hz}$ is associated more with motor cortical function. Slobounov's [30]-[32] recent findings on gamma range $(40 \mathrm{~Hz})$ might be used to discriminate tasks involving visual and vestibular cues.

Along with Sakakibara [17] and Rosenblum [18], Slobounov's [31] work on correlating EEG with posturography to understand human posture control form the impetus for this work. There is a strong suggestion that simultaneous measurement with EEG and stabilograms could potentially be applied to develop a diagnostic tool for neurological pathologies. Additionally, such a study could contribute to providing insights on self-motion perception in virtual environments.

\section{METHODOLOGY}

A postographic analysis under quiet stance is applied to determine the centre of pressure (COP) of the test subject in both the $\mathrm{x}$ - and $\mathrm{y}$-direction, which typically correspond to the medial lateral (ML) and the anterior-posterior (AP) directions respectively. Normally, a human slightly sways in anteriorposterior and lateral directions when standing.

COP (Fig. 1a) is calculated by applying the momentum equation around each sensor (Fig. 1a). By averaging the forces the coordinates of the COP are:

$$
\begin{aligned}
& x=\frac{L \cdot\left(\frac{S_{3}+S_{4}}{W}+\left(1-\frac{S_{1}+S_{2}}{W}\right)\right)}{2} \\
& \mathrm{y}=\frac{L \cdot\left(\frac{\mathrm{S}_{1}+\mathrm{S}_{4}}{\mathrm{~W}}+\left(1-\frac{\mathrm{S}_{2}+\mathrm{S}_{3}}{\mathrm{~W}}\right)\right)}{2}
\end{aligned}
$$

$\mathrm{S}_{\mathrm{i}}$ denotes the force (exerted by the weight of the participant) measured by the sensor $\mathrm{i}$ and $\mathrm{W}=\sum_{\mathrm{i}=1}^{4} \mathrm{~S}_{\mathrm{i}}$, the total force. $\mathrm{L}$ represents the distance between force plate sensor centres, which in this case is $41.88 \mathrm{~mm}$ square.

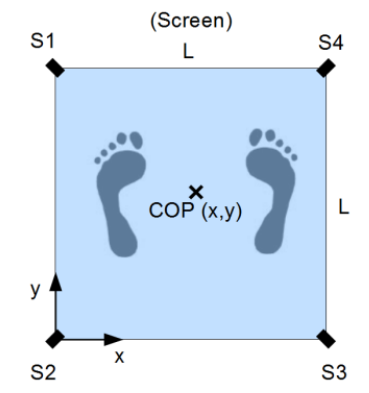

(a)

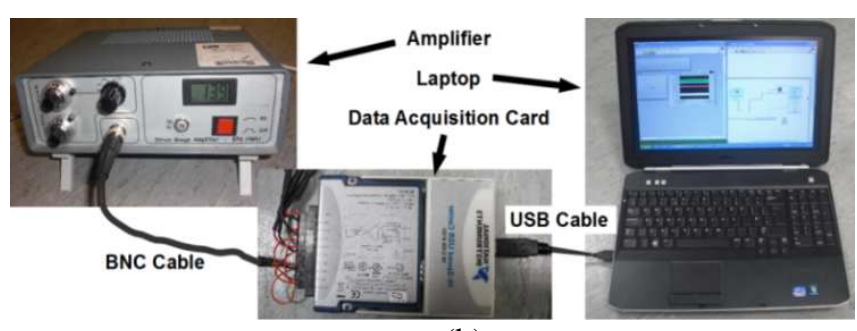

(b)

Fig. 1 (a) Centre of pressure (b) Data acquisition system.

Data acquisition (Fig. 1b) is made up of two modules; a Cseries Hi-speed USB Module Carrier NI USB-9162; and a 100 $\mathrm{kS} / \mathrm{s} / \mathrm{ch}, 16$-Bit, $\pm 10 \mathrm{~V}$ Simultaneous Analog Input Module NI 9215. The reading and the saving of the data is through Labview 2009. The sampling rate has been set at $512 \mathrm{~Hz}$, the same rate as will be used for EEG recordings. Each sensor was calibrated to $800 \mathrm{~N}$ with an Instron machine. Static and dynamic loading with known weights was used to calibrate the force plate to a displacement accuracy of $0.5 \mathrm{~mm}$. 
A NeXus-32 (from Mind Media) multi-modal, multichannel physiological monitoring device is used to acquire EEG signals. This activity measures the electrical potential across specific points on the 10-20 system. Previous investigations [33] have examined the posterior, central and anterior areas, and the left, central and right areas for specific properties. This implies that there is still uncertainty where in the brain the main characteristics of balance occur. Here, all channels will be recorded and different combination of these channels will be compared with the balance data to attempt to discover a relationship between EEG data and balance properties.

Literature suggests the following bandwidths: alpha (8$13 \mathrm{~Hz})$, theta $(3-8 \mathrm{~Hz})$ and beta $(15-25 \mathrm{~Hz})$ as having some influence in standing posture and general orientation. However, research has further indicated that the Gamma band [31] needs to be considered, since it shows a variation for visual recognition of non-stable postures. This means all the bandwidths between $3 \mathrm{~Hz}$ and $50 \mathrm{~Hz}$ will be considered. A bespoke interface was developed to process the EEG signals and the force plate data using MATLAB. The recording and processing appliances are shown in Fig. 2.

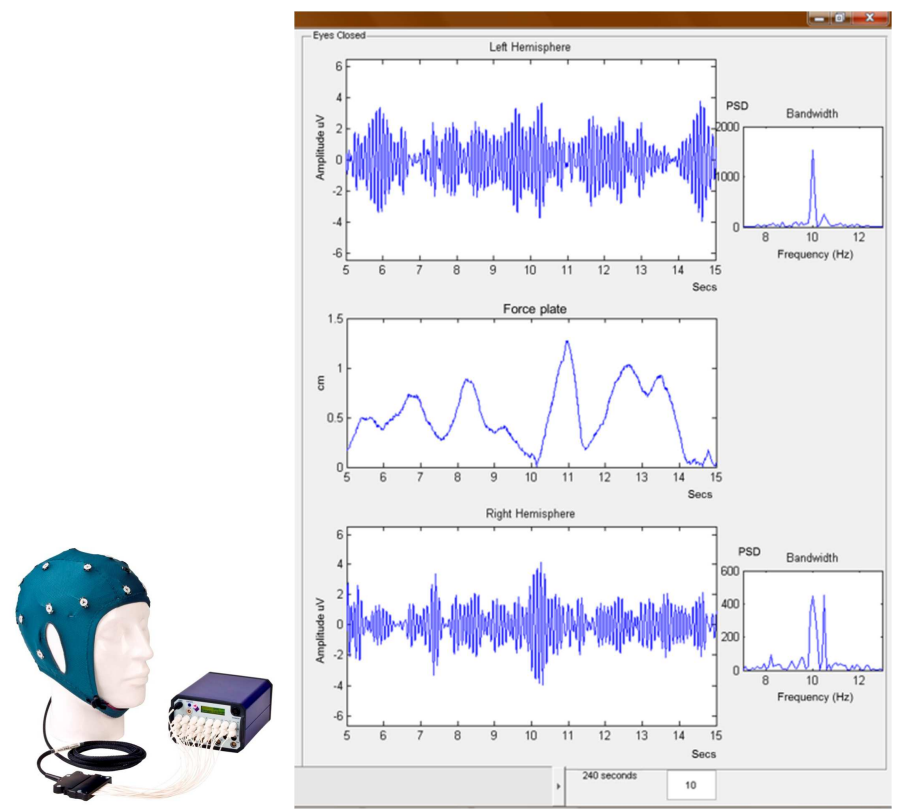

Fig. 2 NeXus-32 unit and GUI to compare force plate data and EEG data.

Briefly, EEG raw signals were filtered using a Butterworth filter in three frequency ranges: low theta $(3-5 \mathrm{~Hz})$, high theta $(6-12 \mathrm{~Hz})$, and gamma $(30-80 \mathrm{~Hz})$. Subsequently, a Fast Fourier Transform (FFT) was used to analyse the frequencies from which power spectrum density (PSD) plots are generated.

\section{IMPLEMENTATION}

A total of 21 participants (20 male, 1 female) volunteered to carry out the experiment. All participants voluntarily disclosed if they had symptoms of dyslexia/dyspraxia
(Developmental Coordination Disorder (DCD)). There are two different conditions of DCD: one is a reading issue, which may or may not be related to balance or motor control. The other is a balance and movement issue. The demographic indicated 5 participants with dyslexia and 16 considered 'healthy'.

The experiment lasts 10 minutes and 20 seconds, which includes 1.5 minutes to explain the procedure to the participant. 4 minutes of standing still with eyes open (EO) were then recorded with the subject looking at a point on a screen at $1.5 \mathrm{~m}$ away. Participants are allowed to blink; the intention of the focus point was to prevent tangential gazing and to keep the head level. It also replicates fixation cues when immersive on a virtual task activity. A one minute rest was then allowed for the participant to relax while still on the force plate in the same position as before. Then 4 minutes were recorded with eyes closed (EC). The experiment is conducted in a low lit room.

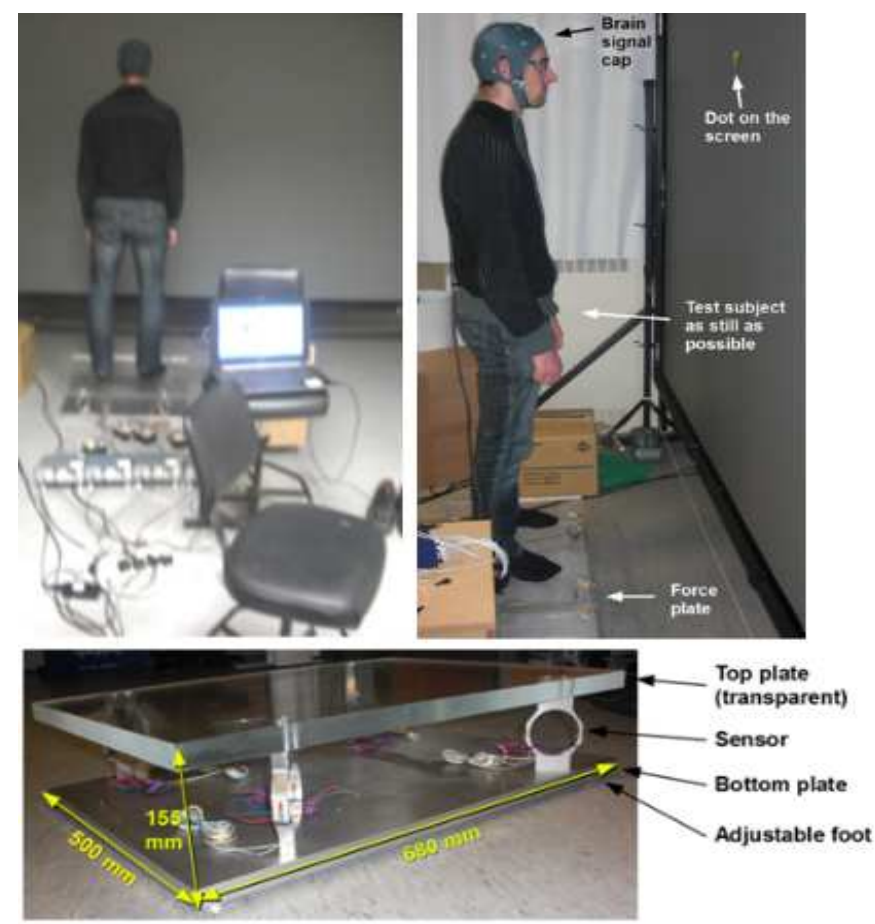

Fig. 3 Experimental set up.

The experimental set up is shown in Fig. 3. The format adopted is similar to Rosenblum's [18] in order to have comparable data for the force plate. The AP directional sway will be denoted as $\mathrm{x}$-displacement and the lateral displacement as y-displacement. Both EEG and force plate signals were recorded at a sampling frequency of $512 \mathrm{~Hz}$. As the goal of the experiment was to measure the unconscious correction of the balance, the participants were asked to stand as still as possible.

\section{RESULTS \& OBSERVATIONS}

Table 1 shows the participant categorisation. The highlighted rows indicate participants whose data had the least amount of noise. Noise here refers to extraneous signals due to EEG sensor movement and/or participant body movements. 


\begin{tabular}{|l|l|l|l|l|l|}
\hline ID & Age & Sex & Dominant Hand & Dominant Foot & Disorder \\
\hline 1 & 21 & M & R & R & NA \\
\hline 2 & 19 & M & L & R & Dyslexia \\
\hline 3 & 21 & M & L & L & Dyslexia/Dyspraxia \\
\hline 4 & 21 & M & R & R & NA \\
\hline 5 & 20 & M & R & R & NA \\
\hline 6 & 21 & M & R & R & NA \\
\hline 7 & 24 & M & R & L & NA \\
\hline 8 & 21 & M & R & R & NA \\
\hline 9 & 21 & M & R & R & Dyslexia \\
\hline 10 & 21 & M & R & R & Dyslexia \\
\hline 11 & 23 & M & R & L & NA \\
\hline 12 & 21 & M & R & R & NA \\
\hline 13 & 22 & M & R & R & NA \\
\hline 14 & 21 & F & R & R & NA \\
\hline 15 & 21 & M & L & R & NA \\
\hline 16 & 21 & M & L & R & NA \\
\hline 17 & 21 & M & R & R & NA \\
\hline 18 & 21 & M & R & R & Dyslexia \\
\hline 19 & 21 & M & R & R & NA \\
\hline 20 & 28 & M & L/R & R & NA \\
\hline 21 & 21 & M & R & L & NA \\
\hline
\end{tabular}

Table 1 Participant demographics and experimental recording.

Fig. 4 shows the shows the power spectral density of the filtered raw EEG signals for each individual EEG channel. The filter applied isolates the frequencies between 7 and $51 \mathrm{~Hz}$ of the full signal. The readings of both participants are mainly located in the alpha bandwidth which aligns well with literature. The peaks in the frontal areas (Fp1\&2, Fz, F4, F5, F7, and F8), the occipital areas (O1 \& O2) as well as some of the other areas, are located around the $10 \mathrm{~Hz}$ frequency and at $50 \mathrm{~Hz}$. The $50 \mathrm{~Hz}$ peak can be explained by the noise infiltrating through the mains which is removed using a bandstop filter. This filtering process is carried out for all other participant data to produce relatively noise free data.

Stabilograms from the force plate measurements are shown in Fig. 5. For each participant, eight plots are drawn for the four minutes EO and for the four minutes EC experiments. The details include: weight distribution as function of the time; the $\mathrm{x}$ and $\mathrm{y}$ coordinates as function of the time; the plan view ( $\mathrm{x}$ against $\mathrm{y}$ plot); the histograms of the $\mathrm{x}$ and $\mathrm{y}$ coordinates with superimposed Gaussian distribution; the PSD plot associated to the $\mathrm{x}$ and $\mathrm{y}$ coordinates.

The weight plot is useful for two reasons; to know the accuracy of the measurements, and to highlight sensor problems. Any change in weight of the same person at the beginning and end of the test voids the results. The plot of the $\mathrm{x}$ coordinate against the time shows the ML displacements of the COP as a function time. The amplitude of these oscillations is in the order of 2 to $4 \mathrm{~mm}$ for both healthy and dyslexic participants. The plot of the y coordinate against the time shows the AP displacements of the COP as a function of time. The amplitude of oscillations is in the order of 10 to $20 \mathrm{~mm}$, with dyslexic subjects being larger overall.

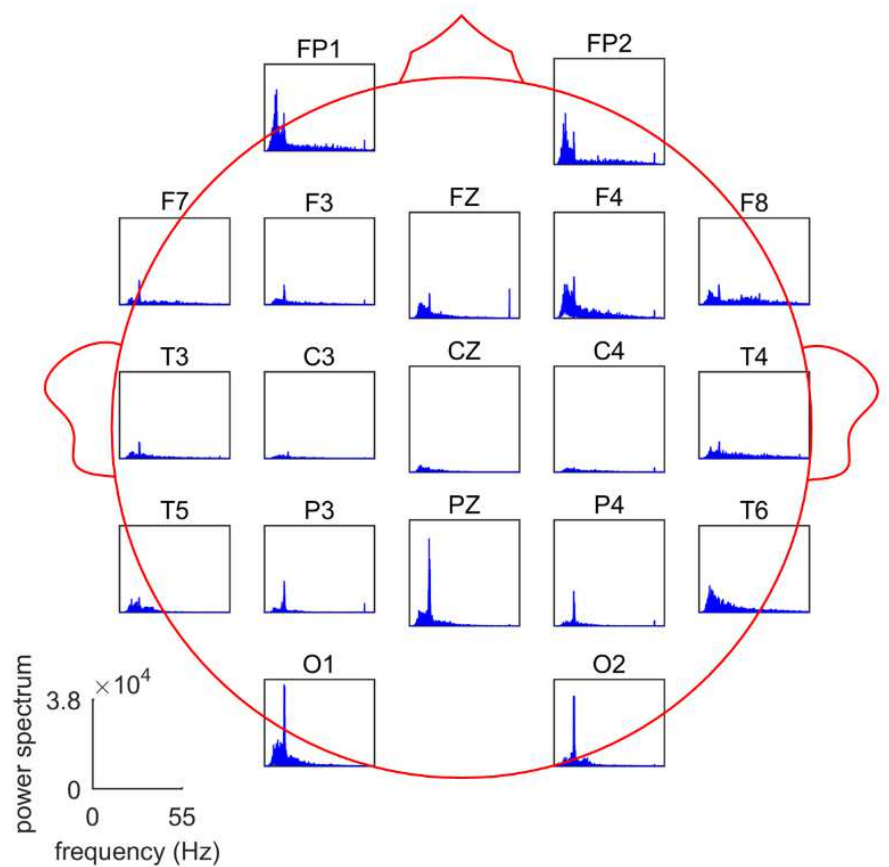

(a) Participant 8 , healthy.

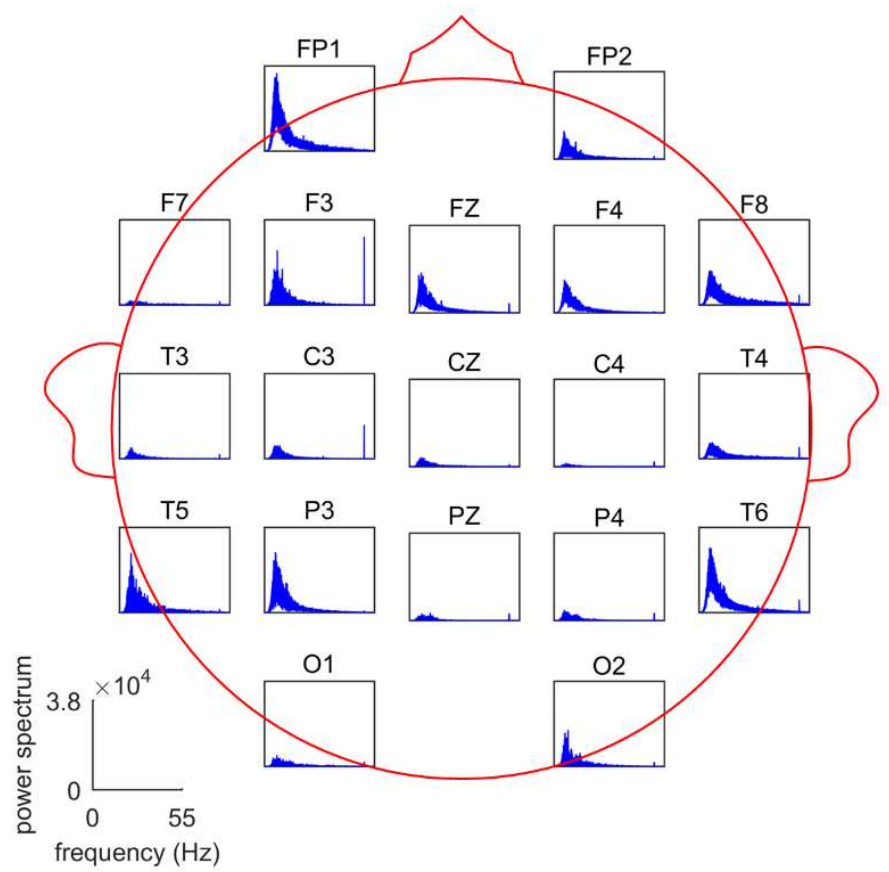

(b) Participant 9, dyslexic.

Fig. 4 Power spectral density plots for eyes open (EO) overlaid on the 10-20 montage. 

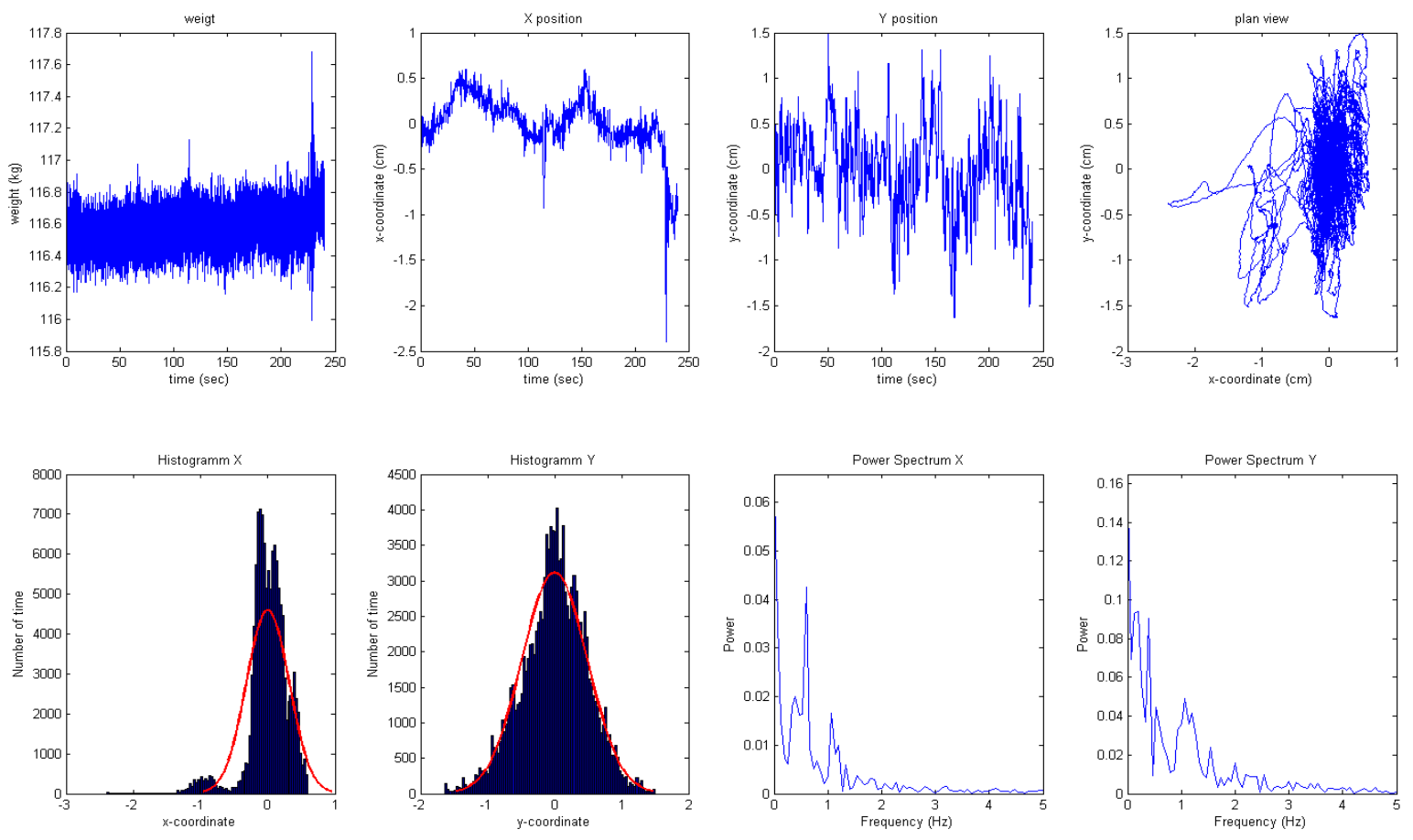

(a) Participant 8, healthy.
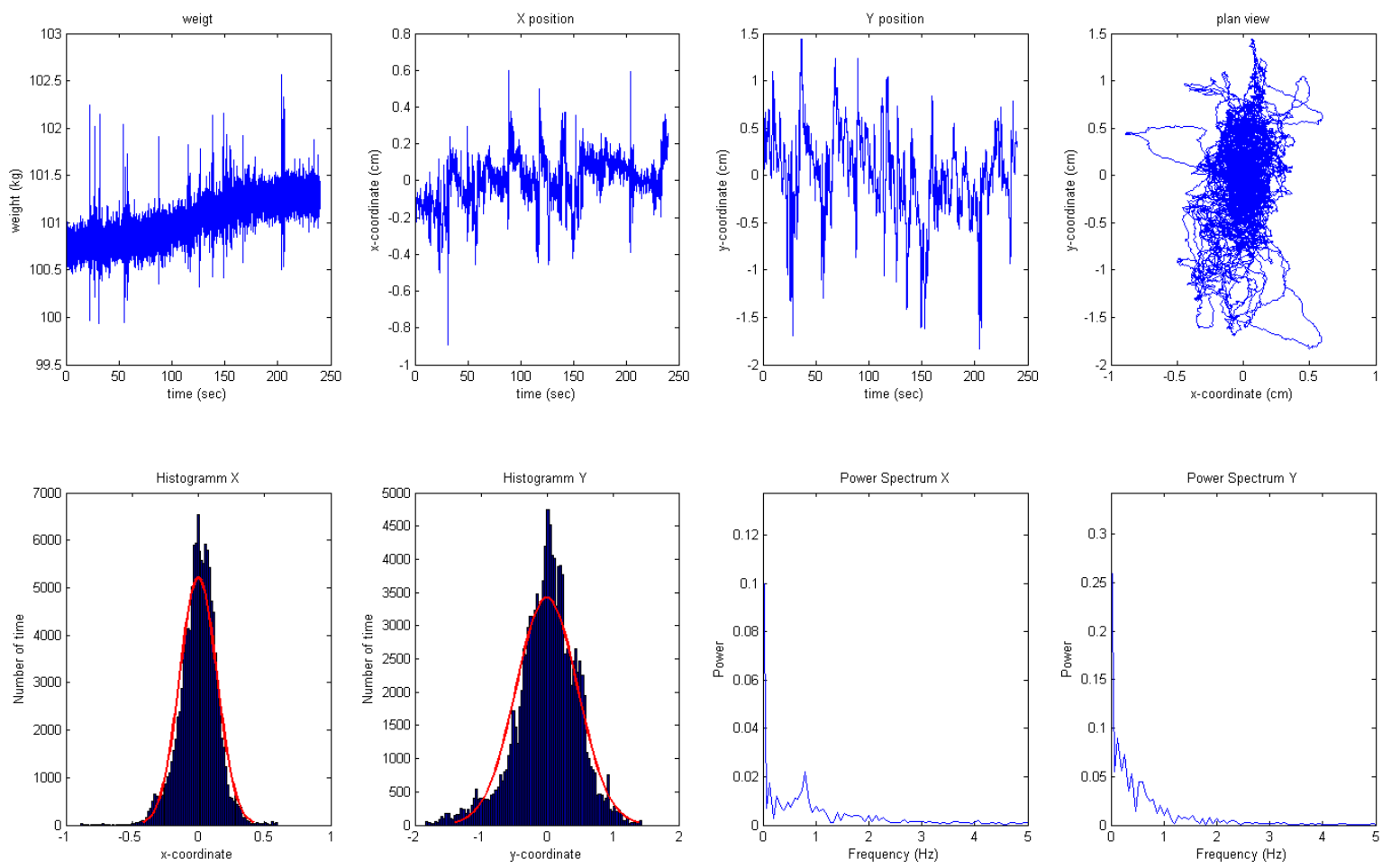

(b) Participant 9, dyslexic.

Fig. 5 Stabilograms of participants 8 (healthy) and 9 (dyslexic). Weight, x-position, y-position, plan view, histograms of $\mathrm{x}$ and $\mathrm{y}$ coordinates, PSD of $x$ and $y$ coordinates for eyes open (EO). 
The plan view in Fig. 5 is the locus of the couple (x,y) in the time. The plan view exhibits a very dense core and some lines that go astray. While interesting, the majority of them are actually errors and correspond to the noise of the force plot. The histograms show the frequency of the COP within small intervals of position respectively in ML and AP. The Gaussian curve establishes the standard deviation and provides a means to quantify how much a person is swaying under quiet stance. The histograms also reveal that participants do not distribute their weight evenly on both legs during the experiment. This slow sway motion appears more often in ML than in the AP direction. The consequence is that the standard deviation is largely only useful to indicate this type of general trend. The PSD can be used to ascertain at which particular frequencies the movements occur.

\section{Observations}

There are some general observations for the posturographic findings: $\mathrm{EO}$ displacements are 2.5 times larger in the AP direction compared to ML; the EC ratio between X- and Ydisplacements is exactly 2 . This is normal, because with the legs slightly apart, a person is very stable in the lateral direction. What is surprising is AP sway under EO which suggests overreaction and/or self-motion perception. Loss of focused on the dot on the screen can result in sudden movements as they try to resume contact with the target. All the other results are consistent with the expectations.

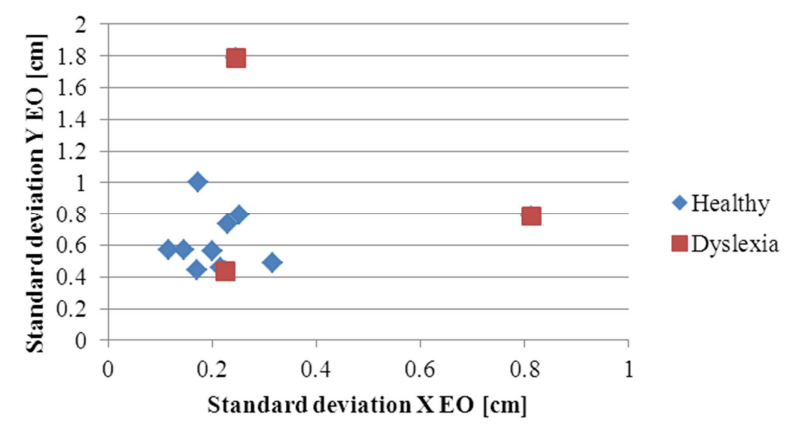

(a) Eyes open (EO)

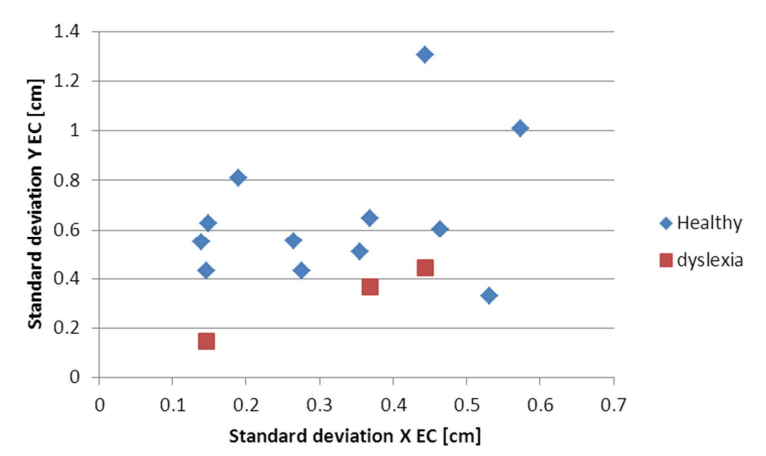

(b) Eyes closed (EC)

Fig. 6 Weight vs. COP. Standard deviation in the $\mathrm{x}$ direction against the y direction with (a) EO and (b) EC.
To understand if weight influenced COP displacement between healthy participants and those with dyslexia a plot of frequency against the standard deviation for $\mathrm{x}$ and $\mathrm{y}$ directions with EO and EC were drawn (Fig. 6). The results suggest a significant difference between healthy and dyslexic subjects. It should be noted that the sample size is small and as such it is statistically not enough to draw conclusions. However, it shows such plots could highlight common pathomechanic characteristics.

The dissymmetry of the histograms could potentially be a symptom of the neurological pathologies too. One way to quantify the dissymmetry is to calculate the median. If the coordinates of the COP were set to zero, and if the histogram is symmetric, the median should be zero. To compare the median between the different participants, it is important to compute a dimensionless number by dividing it by the standard deviation. The plot of this dissymmetry index of the x-histogram against the y-histogram is shown in Fig. 7. The difference though is less significant compared to Fig. 6.

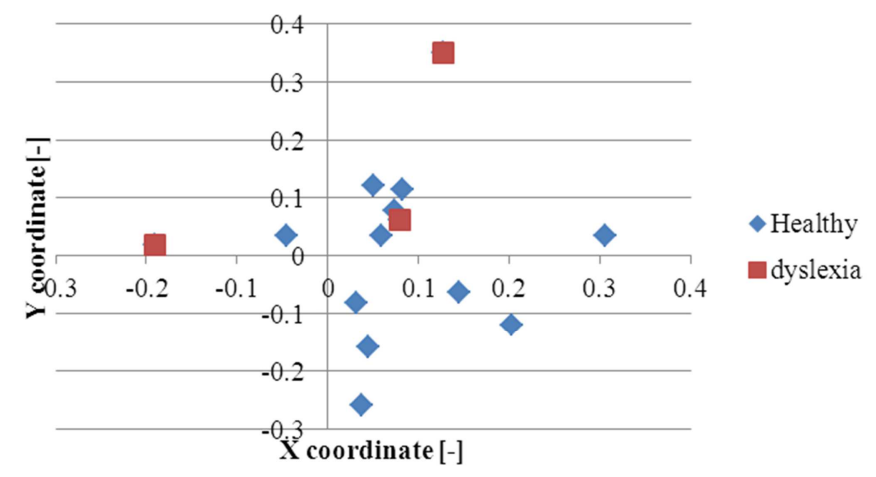

Fig. 7 COP dissymmetry index in $x$ against y coordinate.

Analysing the EEG data on its own, a trend was found. The eyes closed experimentation shows the majority of activity for all participants is located in the frontal region (FP1\&2, F4, 5, 7 and 8), and $\mathrm{O} 1, \mathrm{O} 2, \mathrm{~T} 5$ and $\mathrm{T} 6$. The main difference between the two experiments is the relative increase in alpha activity and the decrease in the higher frequencies.

\section{DISCUSSION}

Neuro-psychophysiological studies suggest the visualvestibular system play a major role in self-motion perception. What is unclear is whether postural sway is linked to selfmotion perception or caused by inputs from other sensory modalities involved in self-motion perception. Virtual environments can visually induce self-motion which cause simulator sickness or could conversely contribute to 'presence'. The discussion here pertains to how the present work could be used with virtual environments to research the visual-vestibular system to identify the onset or likelihood of degenerative pathologies.

Thus far, a direct correlation between the brain signal and the force plate measurements are inconclusive. The experiment 
is limited to a static visual cue. Other than the sample size being small, the frequencies of interest to neurological studies, e.g. EEG analysis, are between 8 and $20 \mathrm{~Hz}$. For the force plate, the PSD shows that the significant movement in the $\mathrm{x}$ and $\mathrm{y}$ directions occur at frequencies lower than $1 \mathrm{~Hz}$. However, the findings indicate that sway can be observed with a combination of force-plate and surface EEG data to represent cortical activity pattern. This is encouraging since meaningful representation of the pathomechanics could be extracted.

More recently, various postural stability measures were recorded using a Wii balance board. This unpublished study ${ }^{1}$ was based on the paradigm a Wii balance board being effectively used a portable balance plate [34]. Results indicate that the Autism Spectrum Disorder (ASD) treatment group showed specific improvements to their balance relative to the ASD wait-list control group - after receiving a programme of skiing. However, the results need to be taken with caution given the relatively small size and lack of a typically developing matched comparison group. Nevertheless, these ASD studies show the utility of using commercial-of-the-shelf gaming hardware such as the Wii board as a portable balance plate for this population.

Under natural conditions with eyes open, active and passive body motion is adequately perceived, even under low light condition. However, with eyes closed the deficiencies of the visual-vestibular system become more apparent. This is expected and is evident in Fig. 6. AP sway under EO suggests the sensation of self-motion, which can affect postural balance. A post experimental interview indicated 7 healthy and 2 dyslexic participants were conscious of self-motion during EO test. It was felt that focusing on the dot caused an illusionary effect. Post EO and EC experiments, 4 healthy and 1 dyslexic participant reported that no discomfort was felt upon dismounting the force plate.

This finding suggests that fidelity in terms of accuracy and realism in the virtual entity representation may not be critical. Rather the virtual environment can be used to create visualvestibular inferences based upon the conscious or unconscious assumption of a stable environment. This could then be used to study or identify abnormal vestibular-ocular reflex movements associated with inner ear pathology or rehabilitation to improve gaze stabilization, tracking, scanning and eye-head coordination.

\section{CONCLUSION}

This investigation set out to establish the possible existence of a relationship between postural sway and EEG data. The results however do not show a definite relationship in a quiet standing still position.

Contrary to research in this field, the general trend in EEG data was found to be located in the occipital lobes. This was not expected since the occipital area does not influence balance

${ }^{1}$ McKennna, P. E., Buckingham, G., Anderson, G., Johanssen, L., Argyropoulos, I., \& Rajendran, G. (unpublished). Does skiing improve children with ASD's balance: a pilot study. according to the literature described. One hypothesis here is that the vestibular system is more dominant compared to somatomotor and somatosensory system. In future these areas will be investigated to see whether they hold any trait with the balance data. The shift in frequency into a higher alpha power spectrum, and a lower power spectrum for the higher frequencies confirms what the literature discussed. Since the alpha bandwidth and lower frequencies are associated with relaxed thinking and sleep, the closing of eyes would initiate the feeling of sleep, where the brain becomes more active in the lower frequencies. Some of the participants commented on a loss of coordination in the last minutes of the experiment. The trend appears to be that after 2 minutes of standing still with eyes closed, the visual feedback is lost, and an increased loss of coordination occurs. Further investigation in the change in EEG readings between the first two minutes of the eyes closed section and the last two minutes will be conducted.

Body posture can provide the first insight into the health of the human motor control system as it continuously acts to balance an inherently unstable body system. Because force platforms can conveniently and precisely produce sway and other motor related time series, this approach to measuring body movements can be advantageous when fast, unobtrusive, and automated evaluation of posturographic information is needed. Force plate records, coupled with classifier analysis, could provide a more sensitive, automated indicator of postural correlates of deception or, indeed, any other cognitive state associated with postural changes. Though the study is limited, it indicates that perception of sway may come from signals related to position, velocity or acceleration, and each sensory system could provide different information about these parameters.

This study is unique in that, unlike most other posturographic and neuronal studies, its aim was not to characterise postural instability. This study exhibits promise for future studies/applications in which a quick, automated classification of body movements relating to emotional state or gestural movement could be identified. It shows that $\mathrm{COP}$ data can be used in order to identify relatively complex movements in a reliable manner. This study has great potential to be expanded upon in other non-traditional posturographic studies such as deception detection.

\section{ACKNOWLEDGMENTS}

The work presented herein is partially funded under the European H2020 Programme BEACONING project, Grant Agreement nr. 687676. The authors would also like to offer their gratitude to G. Frossard and D. van Dieten for their involvement in the research.

\section{REFERENCES}

[1] Kelso, JAS., 1998. From Bernstein's physiology of activity to coordination dynamics. In M. L. Latash (Ed.), Progress in motor control, p.203-19. 
[2] By1 NN. Spatial orientation to gravity and implications for balance training. Orthop Phys Therap Cfin North Am. 1992; 1:207-42.

[3] Baddeley, AD., 1986, "Working Memory", Oxford, Oxford Clarendon Press.

[4] Jeka, JJ., Lackner, JR. The role of haptic cues from rough and slippery surfaces in human postural control. Experimental Brain Research. 1995;103:267-76.

[5] Thelen, E. 1990. Coupling perception and action in the development of skill: A dynamic approach. In H. Bloch \& B. I. Bertenthal (Eds.), Sensory-motor organization and development in infancy and early childhood, p. 39-56.

[6] Zanone, PG., Kelso, JAS. The coordination dynamics oflearning and transfer: Collective and component levels. J. of Experimental Psychology: Human Perception and Performance. 1997;23:1-27.

[7] Woods, RJ., Wilcox, T. Posture Support Improves Object Individuation in Infants. Dev. Psychol. 2012.

[8] Pavol, MJ. Detecting and Understanding Differences in Postural Sway. Focus on "A New Interpretation of Spontaneous Sway Measures Based on a Simple Model of Human Postural Control”. J Neurophysiol. 2005;93:20-1.

[9] Lajoie Y, Teasdale N, Bard C, Fleury M: Attentional demands for static and dynamic equilibrium. Exp Brain Res 1993, 97:139-144.

[10] Andersson G., Hagman J., Talianzadeh R., Svedberg A., Larsen HC. Effect of cognitive load on postural control. Brain Research Bulletin. 2002;58(1): 135-9

[11]Lacour M, Bernard-Demanze L, Dumitrescu M: Posture control, aging, and attention resources: Models and posture-analysis methods. Clin. Neurophysiol 2008, 38:411-21.

[12] Fitzpatrick, R., Burke, D., Gandevia, SC. Loop gain of reflexes controlling human standing measured with the use of postural and vestibular disturbances. J. of Neurophysiol. 1996; 76(6):3994-4008.

[13] Loram, ID., Lakie M. Direct measurement of human ankle stiffness during quiet standing: the intrinsic mechanical stiffness is insufficient for stability. J. of Physiol. 2000; 545(3):1041-53.

[14] Hase, K. Postural Control for Quiet Standing. The Japanese Association of Rehabilitation Medicine. 2006;43:542-53.

[15] Masani K., Vette AH., Milos, R., Popovic, MR. Controlling balance during quiet standing: Proportional and derivative controller generates preceding motor command to body sway position observed in experiments. Gait \& Posture. 2006; 23:164-72.

[16] Kapoula, Z, Matheron, E., Demule E, Fauvel, C., Bucci, M-P. Postural Control during the Stroop Test in Dyslexic and Non Dyslexic Teenagers. PLoS ONE. 2011; 6(4): e19272.

[17] Sakakibara, A. Balance Ability in Acute Stage Rehabilitation Patients. The Society of Physical Therapy Science. 2008;23(2):319-22.

[18] Rosenblum, M., Firsov, G., Kuuz, R., \& Pompe, B. 1998. Human postural control: Force plate experiments and modelling. Nonlinear analysis of physiological data, p. 283-306.

[19] Visser, JE., Nijhuis, LBO, Janssen, L., Bastiaanse, CM., Borm, GF., Duysens J., Bloem, BR. Dynamic posturography in Parkinson's disease: diagnostic utility of the "first trial effect". Neuroscience. 2010;168(2): 387-94.

[20]Fournier, KA., Kimberg, CI., Radonovich, KJ., Tillman, MD., Chow, JW., Lewis, MH., Bodfish JW., Hass, CJ. Decreased static and dynamic postural control in children with autism spectrum disorders. Gait \& Posture. 2010; 32(1):6-9.

[21] Perlmutter, S., Lin, F., Makhsous, M. Quantitative analysis of static sitting posture in chronic stroke. Gait \& Posture, 2010;32(1):53-6.

[22] Dmitrova, ED., Dubrovinskaya, NV., Lukashevich, IP., Machinskaya, RI., Shklovskii, VM. Features of cerebral support of verbal processes in children with dysgraphia and dyslexia. Human Physiology. 2005;31(2):125-31.

[23] Moe-Nilssen, R., Helbostad, JL., Talcott, JB., \& Toennessen, FE. Balance and gait in children with dyslexia. Experimental brain research; 2003; 150(2):23744.

[24] Nijhuis, LO., Hegeman, J., Bakker, M., Van Meel, M., Bloem, BR., Allum, JHJ. The influence of knee rigidity on balance corrections: a comparison with responses of cerebellar ataxia patients. Experimental Brain Research. 2008; 187(2):181-91.

[25] Allum, JHJ., Honegger, F. Interactions between vestibular and proprioceptive inputs triggering and modulating human balance-correcting responses differ across muscles. Experimental brain research. 1998; 121(4):478-94.

[26] Bensel, CK., Dzendolet, E. Power spectral density analysis of the standing sway of males. Perception \& Psychophysics. 1968; 4(5):285-88.

[27] Jo, S., Massaquoi, SG. A model of cerebellum stabilized and scheduled hybrid long-loop control of upright balance. Biological cybernetics. 2004; 91(3):188-202.

[28] Krause, G., Ullsperger, P., Beyer, L., Gille, H-G. Changes in EEG power density spectrum during static muscle work. Eur. J. Appl. Physiol. 1983;51:61-8.

[29] Hari, R., Salmelin, R. Human cortical oscillations: a neuromagnetic view through the skull. Trends in neurosciences. 1997; 20(1):44-49.

[30] Slobounov, S., Tutwiler, R., Slobounova, E., Rearick, M., Ray, W. Human oscillatory brain activity within gamma band $(30-50 \mathrm{~Hz})$ induced by visual recognition of nonstable postures. Cognitive Brain Research.2000; 9(2):17792.

[31] Slobounov, S., Hallett, M., Stanhope, S., Shibasaki, H. Role of cerebral cortex in human postural control: an EEG study. Clinical neurophysiology. 2005; 116(2):315-23.

[32] Slobounov S, Sebastianelli W, Hallet, M.. Residual brain dysfunction observed one year post-mild traumatic brain injury: combined EEG and balance study. Clin Neurophysiol. 2012; 123(9):1755-61. 
[33] Thompson, J. EEG changes and balance deficits following concussion: one piece of the puzzle. Foundations of SportRelated Brain Injuries. 2006; 4(4):341-74.

[34] Travers, B. G., Powell, P. S., Klinger, L. G., \& Klinger, M. R. Motor Difficulties in Autism Spectrum Disorder: Linking Symptom Severity and Postural Stability. Journal of Autism and Developmental Disorders, 2013; 43(7): 1568-1583. 